\title{
Minimum BER Criterion Based Robust Blind Separation for MIMO Systems
}

\author{
Zhongqiang Luo, Wei Zhang, Lidong Zhu and Chengjie Li
}

\begin{abstract}
In this paper, a robust blind source separation (BSS) algorithm is investigated based on a new cost function for noise suppression. This new cost function is established according to the criterion of minimum bit error rate (BER) incorporated into maximum likelihood (ML) principle based independent component analysis (ICA). With the help of natural gradient search, the blind separation work is carried out through optimizing this constructed cost function. Simulation results and analysis corroborate that the proposed blind separation algorithm can realize better performance in speed of convergence and separation accuracy as opposed to the conventional ML-based BSS.
\end{abstract}

Index Terms-Blind Source Separation; Cost Function; Bit Error Rate; Maximum Likelihood; Natural Gradient

\section{INTRODUCTION}

In the past few years, as a paradigm of unsupervised learning in machine learning, blind source separation (BSS) has played an increasingly important role in wireless communication systems for performance enhancement and intelligent information processing [1-14]. It contributes significantly to achieve high spectral efficiency, adaptive signal processing and anti-interference requirements due to its blind feature and statistical information utilization. By virtue of BSS technique, on the one hand, frequently used pilot sequences can be eliminated for enhancing spectral efficiency. On the other hand, it can improve the capacity of the source recovery and resist unpredictable interference in spite of little prior information acquired in advance. In wireless communication systems, a number of received models can be structured as a BSS framework, such as CDMA (code division multiple access) [4-6], OFDM (orthogonal frequency division multiplexing) [7-10] and MIMO (Multiple Input Multiple Output) [11-14], and so on. Generally speaking, those received models can be considered as mixtures of independent source and unknown channel. The expected signals can be

Zhongqiang Luo is with the Artificial Intelligence of Key Laboratory of Sichuan Province, Sichuan University of Science and Engineering, Yibin, Sichuan, China, e-mail: zhongqiangluo@gmail.com.

Wei Zhang is with the with the Artificial Intelligence of Key Laboratory of Sichuan Province, Sichuan University of Science and Engineering, Yibin, Sichuan, China

Lidong Zhu is the National Key Laboratory of Science and Technology on Communications, University of Electronic Science and Technology of China, Chengdu, Sichuan, China, e-mail: zld@uestc.edu.cn

Chengjie Li is the School of Computer Science and Technology, Southwest Minzu University, Chengdu, Sichuan, China, e-mail: junhongabc@126.com. separated or extracted from the received mixed signals by using the independent component analysis (ICA) algorithm based BSS technique.

In a general way, the ICA algorithms are composed of two steps. First, the cost function is built based on an independent principle. Second, the cost function is optimized for blind separation. Therefore, it is vital for constructing the cost function and implementing optimizing scheme. There are three popular independent principles based cost function, which includes maximum likelihood (ML), minimum mutual information (MMI) and non-Gaussian maximization $[1,3]$, respectively. So far, there has been proposed some famous algorithms based on those independent principles, such as FastICA, JADE, Infomax, and so on. Those algorithms are always directly used to carry out blind separation work in the communication system. They always take no account of the performance criterion of the communication system. In fact, those ignored criteria may be combined with independent principles based cost function to propose a more suitable algorithm for executing blind separation of communication mixed signals.

Taking into account of the communication system, the bit error rate (BER) is a significant performance criterion. In this paper, the idea of a minimum BER criterion incorporated into ML or MMI principle is motivated to build the cost function, and then the natural gradient is used to optimize the built new cost function. Simulation results show the proposed new cost function based blind separation algorithm can lead to better performance in speed of convergence and separation accuracy compared with the original one.

The remainder of the paper is organized as follows. In the Section II, the system model of blind source separation is reviewed. The new cost function of ICA and the proposed blind separation algorithm are both described in Section III. Simulation results and discussion are presented in Section IV. Section V draws the conclusions.

\section{SYSTEM MODEL}

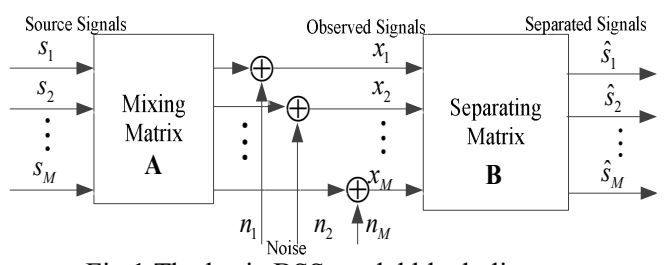

Fig.1 The basic BSS model block diagram 
In this section, the basic BSS model is reviewed. As shown in Fig. 1, the BSS model has a close relationship to MIMO system $[1,14]$. Considering the determined BSS model, that is to say, the number of transmitting antennas and receiving antenna is the same in MIMO system. The mutually independent source vector is denoted as $\mathbf{S}=\left(s_{1}, s_{2}, \ldots s_{M}\right)^{T}$. The mixing matrix is $\mathbf{A}$, which describes a MIMO channel condition. $\mathbf{n}=\left(n_{1}, n_{2}, \ldots, n_{M}\right)^{T}$ is the noise vectors. The observed mixed signal is $\mathbf{x}=\left(x_{1}, x_{2}, \ldots, x_{M}\right)^{T}$, in other words, the received signals in MIMO. The received mixed signals can be described as follows,

$$
\mathbf{x}=\mathbf{A} \mathbf{s}+\mathbf{n}
$$

The goal of BSS is to separate or extract source signals only from observed mixed signals. The source signal estimation can be obtained after the separating operation is executed,

$$
\begin{aligned}
\hat{\mathbf{s}} & =\mathbf{B A s}+\mathbf{B n} \\
& =\mathbf{s}+\mathbf{B n}
\end{aligned}
$$

Ideally, $\mathbf{C}=\mathbf{B} \mathbf{A}$ is an identity matrix, i.e., the separating matrix $\mathbf{B}$ is the inverse of the mixing matrix. In fact, the matrix $\mathbf{C}$ is a generalized permutation matrix due to inherent indeterminacy in BSS. However, this problem has no effect into the separation work.

\section{NEW COST FUNCTION FOR BSS}

\section{A. ML principle based cost function}

The cost function of the ICA problem is usually derived via the maximum likelihood (ML) approach under the independence assumption. Suppose that sources $\mathbf{S}$ are independent with marginal distribution $f_{i}\left(s_{i}\right)$.

$$
f_{\mathbf{s}}(\mathbf{s})=\prod_{i}^{M} f_{s_{i}}\left(s_{i}\right)
$$

In the linear model, $\mathbf{x}=\mathbf{A s}$, the joint density of the observation vector is related to the joint density of the source vector as follows:

$$
f_{\mathbf{x}}(\mathbf{x})=\frac{1}{|\operatorname{det} \mathbf{A}|} f_{\mathbf{s}}\left(\mathbf{A}^{-1} \mathbf{x}\right)=\left|\operatorname{det} \mathbf{A}^{-1}\right| f_{\mathbf{s}}\left(\mathbf{A}^{-1} \mathbf{x}\right)
$$

Then our goal is to find a maximum likelihood estimation of $\mathbf{A}$ (or $\mathbf{B}$ where $\mathbf{B}=\mathbf{A}^{-1}$ ) to maximize (4). Noting that $\mathbf{y}=\mathbf{A}^{-1} \mathbf{x}=\mathbf{B} \mathbf{x}$, the ML cost function can be derived from the log likelihood of (4) as

$$
\log f_{\mathbf{x}}(\mathbf{x})=-\log |\operatorname{det} \mathbf{A}|+\log f_{\mathbf{s}}\left(\mathbf{A}^{-1} \mathbf{x}\right)
$$

which can be also written as

$$
\log f_{\mathbf{x}}(\mathbf{x})=\log |\operatorname{det} \mathbf{B}|+\log f_{\mathbf{y}}(\mathbf{y})
$$

replaced by a hypothesized distribution $f_{\mathbf{y}}(\mathbf{y})$. Since sources are assumed to be statistically independent, the cost function is written as

$$
J(\mathbf{B})=-\log |\operatorname{det} \mathbf{B}|-\sum_{i=1}^{M} \log f_{y_{i}}\left(y_{i}\right)
$$

The separating matrix $\mathbf{B}$ is determined by

$$
\hat{\mathbf{B}}=\underset{\mathbf{B}}{\arg \min }\left\{-\log |\operatorname{det} \mathbf{B}|-\sum_{i=1}^{M} \log f_{y_{i}}\left(y_{i}\right)\right\}
$$

\section{B. Minimum BER constrained ML principle based cost function}

In this subsection, the minimum BER criterion is derived firstly. Then the minimum BER criterion incorporated into ML principle based cost function is built. The BSS problem in MIMO is a blind equalization one. Taking into account communication signals in a MIMO system model, the transmitted symbols are equiprobable antipodal symbols (i.e., \pm 1 , BSPK) uncorrelated with each other, i.e.,

$$
E\left\{\mathbf{s s}^{T}\right\}=\mathbf{I}
$$

The antipodal assumption is made for simplicity, and other constellations can also be used to extend, such as 4-QAM/QPSK. The noise vector $\mathbf{n}$ is zero-mean, white and Gaussian, with covariance matrix

$$
E\left\{\mathbf{n} \mathbf{n}^{T}\right\}=\sigma^{2} \mathbf{I}
$$

When $\mathbf{S}$ is transmitted, $\hat{\mathbf{s}}$, as given by (2), will be the received signal vector. The elements of this vector are then quantized by a threshold detector to obtain $\hat{\mathbf{s}}_{q}$, whose elements will be \pm 1 . The average BER of the detected signal is the average of the probability of error of each element of the block, i.e.,

$$
P_{e}=\frac{1}{M} \sum_{m=1}^{M} P_{e m}
$$

In which $P_{e m}$ denotes the BER of the $m^{\text {th }}$ source symbol. Since the signal power of each data symbol is unity and the covariance matrix of the received noise is $\sigma^{2} \mathbf{B B}^{T}$, by following standard steps, it can be shown that the probability of the $m^{\text {th }}$ source symbol in $\hat{\mathbf{s}}_{q}$ being in error can be written as

$$
P_{e m}=\frac{1}{2} \operatorname{erfc}\left(\frac{1}{\sqrt{2 \sigma^{2}\left[\mathbf{B B}^{T}\right]_{m m}}}\right)
$$

Where, $\operatorname{erfc}(\varsigma) \triangleq(2 / \sqrt{\pi}) \int_{\varsigma}^{\infty} e^{-z^{2}} d z$, and $\left[\mathbf{B B}^{T}\right]_{m m}$ denotes the $(m, m)^{t h}$ element of the matrix $\mathbf{B} \mathbf{B}^{T}$. The term

$\mathbf{y}$ is the estimation of $\mathbf{s}$ with the actual distribution $f_{\mathbf{s}}(\mathbf{s})$ 
$\sigma^{2}\left[\mathbf{B B}^{T}\right]_{m m}$ represents the noise variance in the receiver's estimation of the $m^{\text {th }}$ source symbol of the transmitted signal vector. Substituting (12) into (11), yielding

$$
P_{e}=\frac{1}{2 M} \sum_{m=1}^{M} \operatorname{erfc}\left(\frac{1}{\sqrt{2 \sigma^{2}\left[\mathbf{B B}^{T}\right]_{m m}}}\right)
$$

If we assume $\phi(z)=\operatorname{erfc}\left(1 / \sqrt{2 \sigma^{2} z}\right)$ for $z>0$, then

$$
\frac{d^{2} \phi}{d z^{2}}=\frac{1}{\sqrt{\pi}}\left(2 \sigma^{2}\right)^{-(1 / 2)} \exp \left(-\frac{1}{2 \sigma^{2} z}\right)\left(-\frac{3}{2}+\frac{1}{2 \sigma^{2} z}\right) z^{-(5 / 2)}
$$

Therefore, if $z<1 / 3 \sigma^{2}$, then $d^{2} \phi / d z^{2}>0$. Applying this fact to(13), $\phi\left(\left[\mathbf{B B}^{T}\right]_{m m}\right)$ is a convex function if the noise power $\sigma^{2}$ is less than $1 / 3\left[\mathbf{B B}^{T}\right]_{m m}$. If this condition is satisfied for all $m$ (i.e., if there is sufficiently large SNR at the receiver), the average block BER $P_{e}$ is also convex [15].

Since $P_{e}$ is convex at moderate-to-high SNRs, the Jensen's inequality can be applied to obtain the following lower bound on $P_{e}$ :

$$
\begin{aligned}
P_{e} & =\frac{1}{2 M} \sum_{m=1}^{M} \operatorname{erfc}\left(\frac{1}{\sqrt{2 \sigma^{2}\left[\mathbf{B B}^{T}\right]_{m m}}}\right) \\
& \geq \frac{1}{2} \operatorname{erfc}\left(\frac{1}{\sqrt{\frac{2 \sigma^{2}}{M} \sum_{m=1}^{M}\left[\mathbf{B B}^{T}\right]_{m m}}}\right) \\
& =\frac{1}{2} \operatorname{erfc}\left(\sqrt{\frac{M}{2 \sigma^{2} t r\left(\mathbf{B B}^{T}\right)}}\right)=P_{e, L B}
\end{aligned}
$$

Equality in (15) holds if and only if $\left[\mathbf{B B}^{T}\right]_{m m}$ are equal $\forall m \in[1, M]$. The inequality of (15) is valid only when $P_{e}$ is convex, i.e., when

$$
\left[\mathbf{B B}^{T}\right]_{m m}<\frac{1}{3 \sigma^{2}}, \forall m \in[1, M]
$$

The quantity $P_{e, L B}$ in (15) defines a lower bound on the BER $P_{e}$. Note that since $\operatorname{erfc}(\cdot)$ is a monotonically decreasing function, to minimize $P_{e, L B}$ in (15), we need only minimize $\operatorname{tr}\left(\mathbf{B B}^{T}\right)$. That is to say, the minimum BER criterion can be described as follows:

$$
\begin{gathered}
\min _{\mathbf{B}} \operatorname{tr}\left(\mathbf{B B}^{T}\right) \\
\text { subject to }\left[\mathbf{B B}^{T}\right]_{m m}<\frac{1}{3 \sigma^{2}}
\end{gathered}
$$

Combined with (7), the new cost function with minimum BER criterion can be obtained,

$$
\left\{\begin{array}{l}
\underset{\mathbf{B}}{\arg \min }\left\{-\log |\operatorname{det} \mathbf{B}|-\sum_{i=1}^{M} \log f_{y_{i}}\left(y_{i}\right)\right\} \\
\underset{\mathbf{B}}{\min } \operatorname{tr}\left(\mathbf{B B}^{T}\right) \\
\text { subject to } \operatorname{tr}\left[\mathbf{B B}^{T}\right]<\frac{M}{3 \sigma^{2}}
\end{array}\right.
$$

In order to simplify the above constrained optimization problem (17), considering (7), the new cost function with minimum BER criterion in condition of the moderate-to-high SNRs can be described as a unconstrained optimization problem, i.e.,

$$
\hat{\mathbf{B}}=\underset{\mathbf{B}}{\arg \min }\left\{-\log |\operatorname{det} \mathbf{B}|-\sum_{i=1}^{M} \log f_{y_{i}}\left(y_{i}\right)+\lambda \operatorname{tr}\left(\mathbf{B} \mathbf{B}^{T}\right)\right\}
$$

Where $\lambda$ is a regulation parameter. Then the natural gradient search for optimizing the cost function (18) can be realized for BSS.

\section{Optimizing cost function by natural gradient}

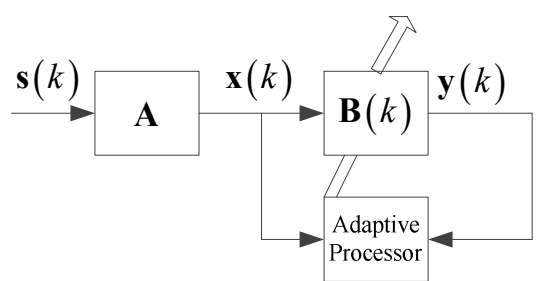

Fig.2 Adaptive processor block diagram

As the previous illustration, the ICA-based blind separation algorithms include a two-step process. The first step is to choose a principle, based on which a cost function is obtained. Next, a suitable method for optimizing the cost function needs to be adopted. In other words, using a cost function converts the blind separation problem into an optimization problem. In this paper, the separation processing is implemented by the adaptive BSS based on the natural gradient for its fast and accurate adaptation behavior. The adaptive processor block diagram for BSS is shown in Fig. 2. For any suitable smooth 
gradient-searchable the cost function $J(\mathbf{B})$, the natural adaptation is defined as:

$$
\mathbf{B} \leftarrow \mathbf{B}-\mu \nabla J(\mathbf{B}) \mathbf{B}^{T} \mathbf{B}
$$

The new built cost function in (18), since the probability density function (PDF) of sources are supposed to be unknown, and $f_{y_{i}}\left(y_{i}\right)$ is also unknown. Therefore, the activation or score function need be used to approximate probability density function of separation source signals.

The function $\varphi_{i}\left(y_{i}\right)$ denotes the activation or score function in ML approach, which is defined as

$$
\varphi_{i}\left(y_{i}\right)=-\frac{d \log f_{y_{i}}\left(y_{i}\right)}{d y_{i}}=-\frac{f_{y_{i}}^{\prime}\left(y_{i}\right)}{f_{y_{i}}\left(y_{i}\right)}
$$

Since sources in digital communication are always subgaussian signal, this activation function can be chose as follows [3],

$$
\varphi_{i}\left(y_{i}\right)=-y_{i}^{3}
$$

Furthermore we can obtain the gradient of the cost function as follows:

$$
\nabla J(\mathbf{B})=\frac{\partial J(\mathbf{B})}{\partial \mathbf{B}}=-\mathbf{B}^{-T}+\boldsymbol{\varphi}(\mathbf{y}) \mathbf{x}^{T}+2 \lambda \mathbf{B}
$$

The natural gradient learning law now yields

$$
\mathbf{B} \leftarrow \mathbf{B}-\mu\left(\mathbf{I}-\boldsymbol{\varphi}(\mathbf{y}) \mathbf{y}^{T}-2 \lambda \mathbf{B} \mathbf{B}^{T}\right) \mathbf{B}
$$

\section{SimUlations AND Discussions}

To demonstrate the effectiveness of the proposed algorithm in this paper, we conduct simulation experiments to evaluate the performance of the proposed ML based cost function with minimum BER criterion by nature gradient (called ML-BER-NG). For comparison, the only ML based cost function by nature gradient (ML-NG) is also illustrated for highlighting the proposed algorithm mechanism by comparative experiments. Considering a MIMO system, the number of transmitting antennas and receiving antennas is 5 , the source symbols are from BPSK, the sample size is 4000 . The mixing matrix is generated randomly. The performance index is cross talk error. The smaller is this performance index, the better performance is acquired. The cross talk error is defined as following[1, 3]

$$
E_{c t}=\sum_{i=1}^{M}\left(\sum_{j=1}^{M} \frac{\left|c_{i j}\right|}{\max _{k}\left|c_{i k}\right|}-1\right)+\sum_{j=1}^{M}\left(\sum_{i=1}^{M} \frac{\left|c_{i j}\right|}{\max _{k}\left|c_{k j}\right|}-1\right)
$$

where $\mathbf{C}=\mathbf{B A}, c_{i j}$ is element in matrix $\mathbf{C}$. The moderate SNR condition is considered and other parameters setting are same for two methods.

The simulation results are demonstrated in Fig. 3 and Fig. 4, respectively. We can conclude that the proposed cost function by nature gradient can lead to better performance in speed of convergence and separation accuracy. In Fig. 3, we can see that the proposed ML-BER-NG algorithm has low cross talk error and fast convergence rate compared with the original ML-NG algorithm. It is noteworthy that the initial value of a separation matrix is randomly generated, so that the number of iterations is a bit larger. However, the computation complexity is low. It takes just 2-4 seconds to achieve the algorithm convergence from time complexity. From Fig. 4, we can see that BER performance of the ML-BER-NG is better than the ML-NG in moderate SNR condition.

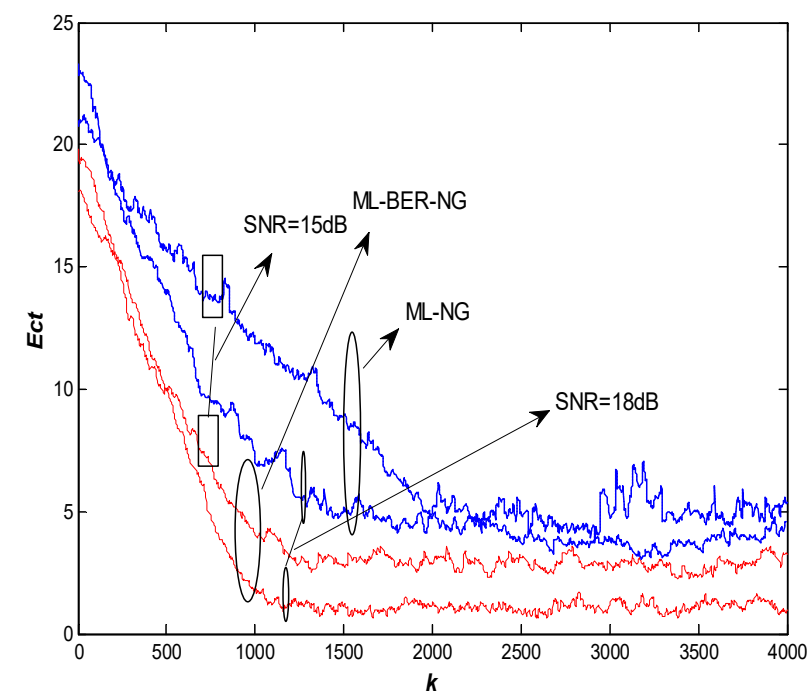

Fig. 3 The cross talk error as a function of iterations

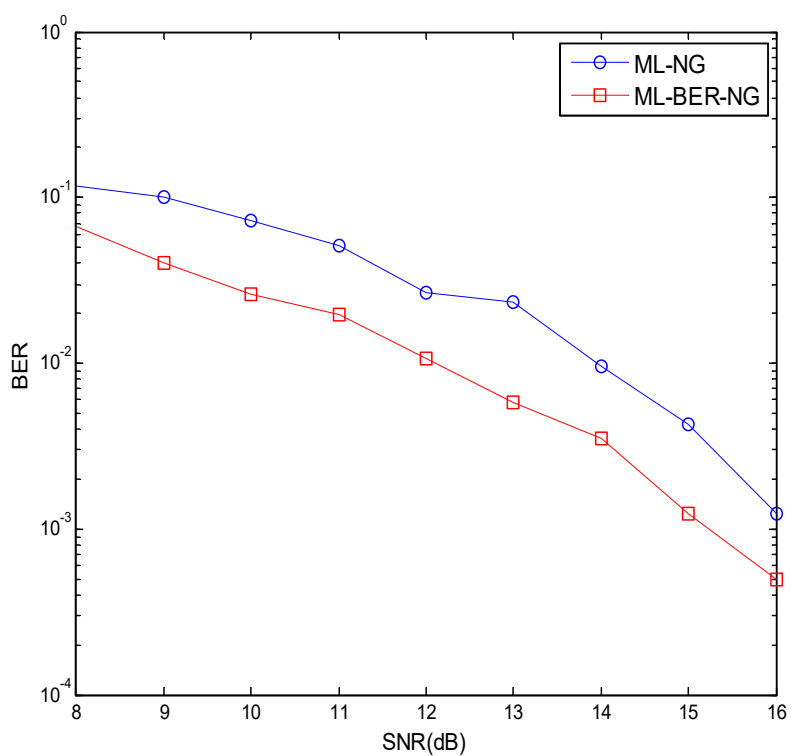

Fig.4 BER performance as a function of SNR

In Fig.5, the further performance comparison of the representative methods is conducted 100 experiments for highlighting the proposed algorithm when the moderate $\mathrm{SNR}=10 \mathrm{~dB}$. In Fig.5(a), the statistical analysis of BER 
performance is given in a boxplot form, and in Fig.5(b) the BER performance with error bar is drawn to exhibit the performance enhancement of the proposed method compared with other representative algorithms. We can safely obtain that the proposed method achieve the performance refinement in contrast with that of some representative BSS methods for MIMO signals detection.

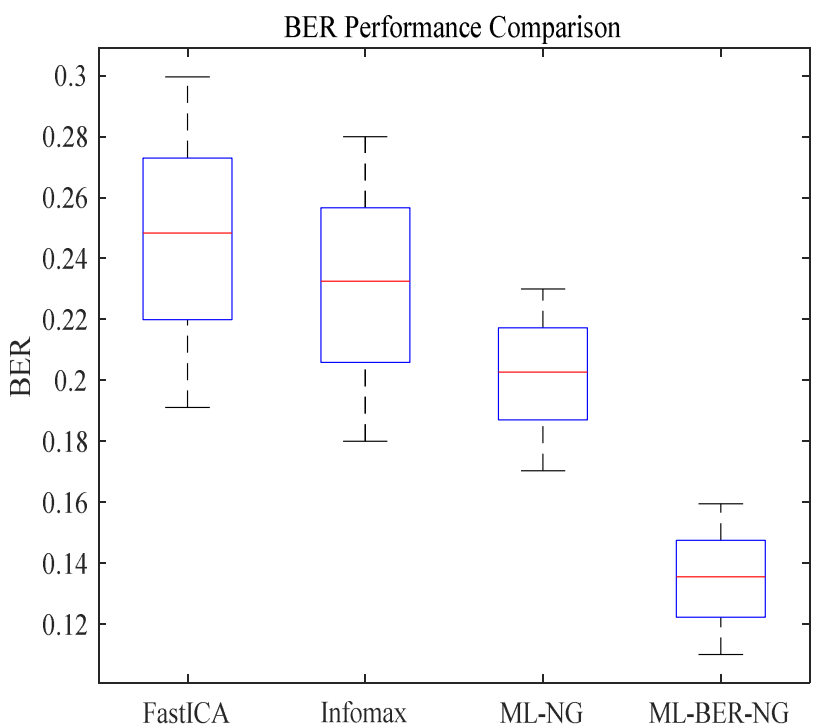

(a)

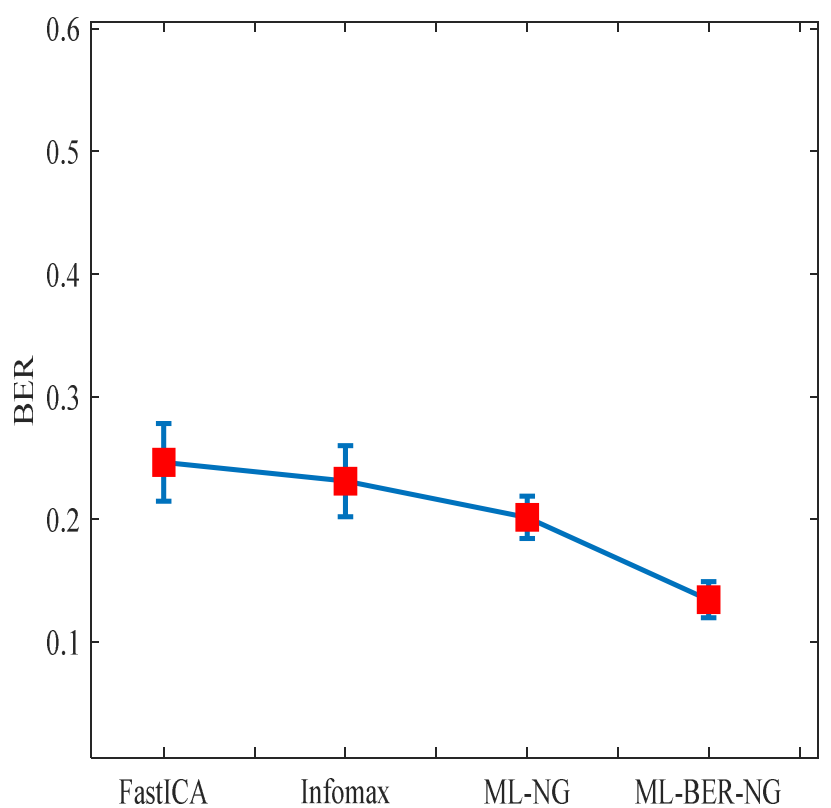

(b)

Fig. 5 BER performance comparisons of different representative methods
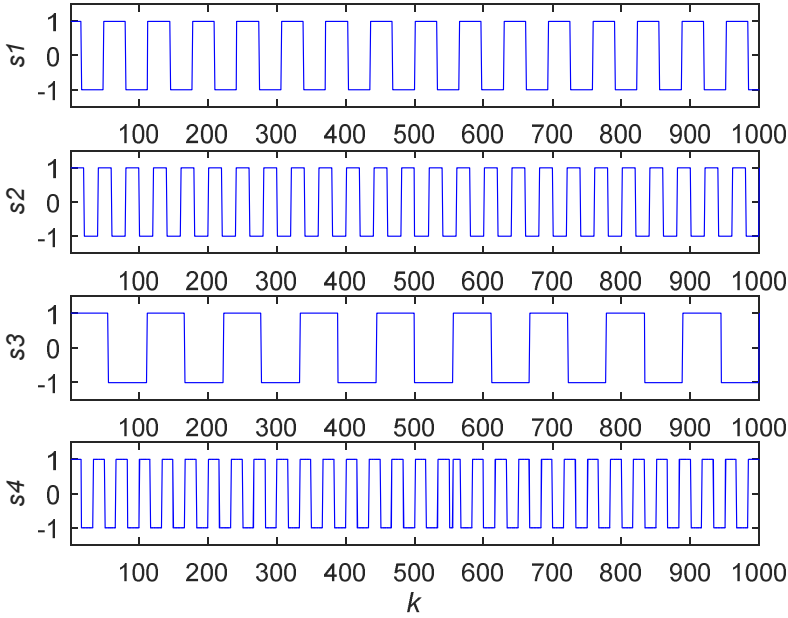

(a) Transmitted signals;
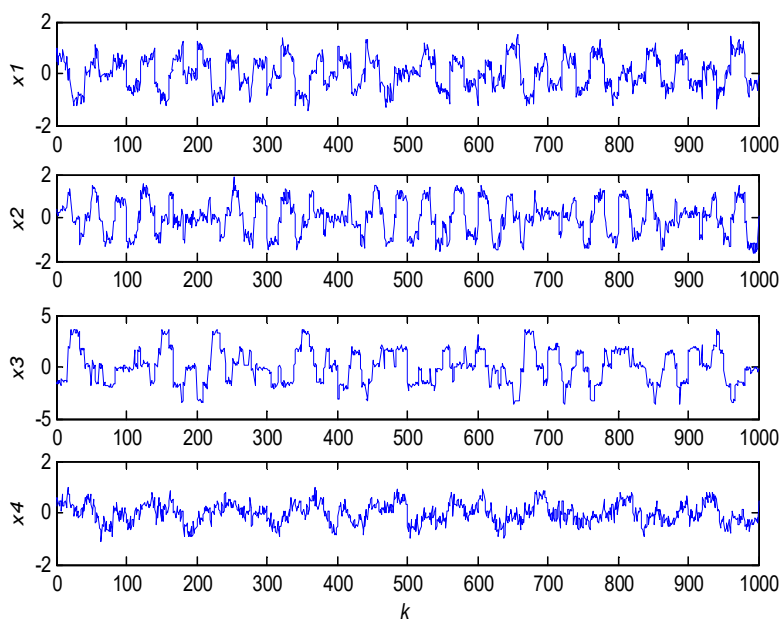

(b) Received mixed signals;
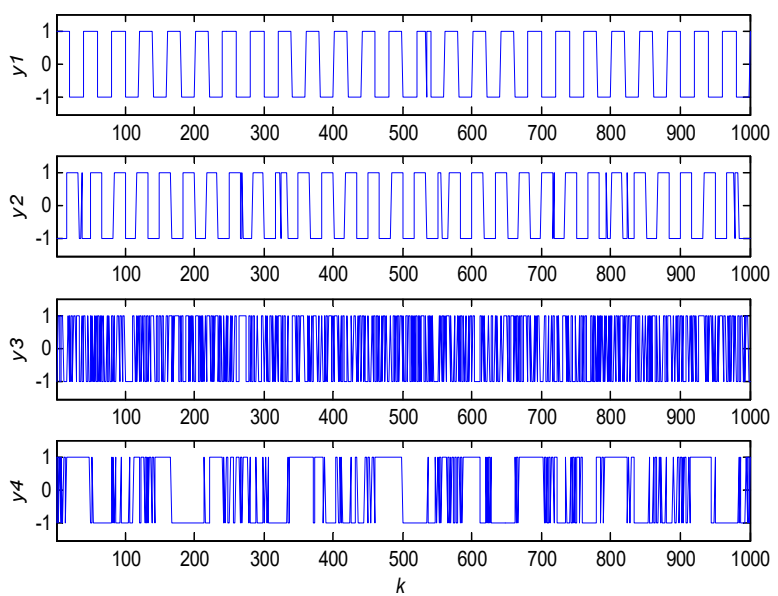

(c) Separated detected signals (ML-NG) 

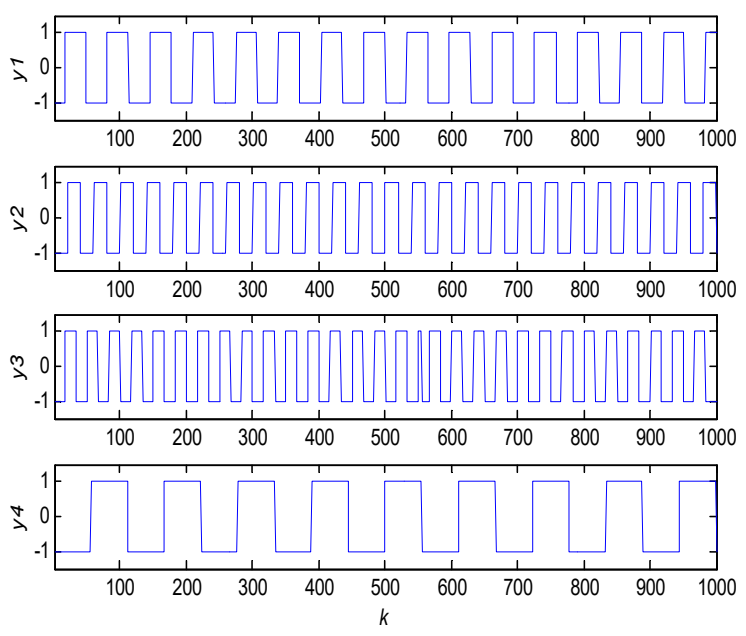

(d) Separated detected signals (ML-BER-NG)

Fig.6 $4 \times 4$ MIMO; (a) Transmitted signals; (b) Received mixed signals; (c) Separated detected signals (ML-NG) (d) Separated detected signals (ML-BER-NG)

In the next simulation, the direct separation graphs are exhibited for illustration. As shown in Fig.6, it shows the results of blind separation of $4 \times 4 \mathrm{MIMO}$ in $\mathrm{SNR}=15 \mathrm{~dB}$. From the separated results of Fig.6, we can verify that the incorporation of minimum BER criterion in BSS improves the separation performance.

Remarks: The ML principle with minimum BER criterion considers the effect of noise term in the model of the cost function. It is fit for communication signals circumstance. The original ML principle neglects the effect of noise. However, noise is inevitable in a wireless communication environment. Furthermore, the computation complexity of the proposed algorithm (ML-BER-NG) is nearly similar to the ML-NG algorithm with low computation.

\section{CONCLUSIONS}

In this paper, a minimum BER criterion is considered in combination with the ML independent principle for blind separation problem in MIMO signal detection. The effect of the noise term is taken into account in the process of constructing cost function. The proposed algorithm can lead to better performance in speed of convergence and separation accuracy in moderate SNR condition. It is strongly encouraged to investigate the constrained cost function for BSS problem in low SNR in the future work. It is promising idea for thinking over other communication performance criteria for developing advanced BSS algorithms.

\section{ACKNOWLEDGMENT}

The paper is supported by Natural Science Foundation of China (No. 61801319, No. 61871422), the Opening Project of Artificial Intelligence Key Laboratory of Sichuan Province (No. 2017RZJ01), the Opening Project of Key Laboratory of
Higher Education of Sichuan Province for Enterprise Informationalization and Internet of Things (No. 2017WZJ01), Sichuan University of Science and Engineering Talent introduction project (No.2017RCL11), the Education Agency Project of Sichuan Province (No.18ZB0419), Education Reform Project of Sichuan University of Science and Engineering(No. JG-1810), and the Major Frontier Project of Science and Technology Plan of Sichuan Province (No. 2018JY0512).

\section{REFERENCES}

[1] Tülay Adali, Simon Haykin, "Adaptive Signal Processing: Next Generation Solution”, Hoboken, NJ: Wiley-IEEE Press, 2010.

[2] Zhongqiang Luo, Chengjie Li, Lidong Zhu, "A Comprehensive Survey on Blind Source Separation for Wireless Adaptive Processing: Principles, Perspectives, Challenges and New Research Directions, " IEEE Access, vol. 6, pp. 1-24, 2018

[3] X. Yu, D. Hu, J. Xu, "Blind Source Separation: Theory and Applications", Singapore: John Wiley \& Sons, 2014.

[4] Jiang Zhang, Hang Zhang and Zhifu Cui, "Dual-antenna-based blind joint hostile jamming cancellation and multi-user detection for uplink of asynchronous direct-sequence code-division multiple access systems," IET Commun., Vol. 7, No. 10, pp. 911-921, 2013

[5] Zhongqiang Luo, Lidong Zhu, "A Charrelation Matrix-Based Blind Adaptive Detector for DS-CDMA Sytsems,"Sensors, vol. 15, no. 8, pp. 20152-68, 2015.

[6] FU Wei-hong, YANG Xiao-niu, LIU Nai-an, "The Multi-User Detection and Chip Sequence Estimation for CDMA System Based on the Blind Source Separation,"ACTA ELECTRONICA SINICA, Vol. 36, No. 7, pp.1319-1323, 2008.

[7] Zhongqiang Luo, Lidong Zhu, Chengjie Li, "Employing ICA for Inter-Carrier Interference Cancellation and Symbol Recovery in OFDM Systems", in Proc. IEEE Global Communications Conference (GLOBECOM 2014), Austin, USA, pp. 3501-3505, Dec. 2014.

[8] Zhongqiang Luo, Lidong Zhu, Chengjie Li, "Independent Component Analysis based Blind Adaptive Interference Reduction and Symbol Recovery for OFDM Systems", China Communications, vol. 13, no.2, pp. 41-54, 2016

[9] M.G.S. Sriyananda, Jyrki Joutsensalo, Timo Hämäläinen, "Blind Source Separation for OFDM with Filtering Colored Noise and Jamming Signal,"Journal of Communications and Networks, Vol. 14, No. 4, pp. 410-417, 2012.

[10] Zhongqiang Luo, Lidong Zhu, Chengjie Li, "Vandermonde Constrained Tensor Decomposition Based Blind Carrier Frequency Synchronization for OFDM Transmissions", Wireless Personal Communications, Vol. 95, No. 3, pp.3499-3508, 2017.

[11] Iman Moazzen, "Array Signal Processing for Beamforming and Blind Source Separation," University of Victoria, Doctoral Dissertation, 2013.

[12] Zhongqiang Luo, Lidong Zhu, Chengjie Li, "Exploiting Large Scale BSS Technique for Source Recovery in Massive MIMO Systems", in Proc. IEEE/CIC ICCC, 2014, pp. 391-395.

[13] Zhongqiang Luo, Chengjie Li, Lidong Zhu, "Robust Blind Separation for MIMO Systems against Channel Mismatch Using Second-Order Cone Programming", China Communications, vol. 14, no. 6, pp. 168-178, 2017.

[14] Zhongqiang Luo, Chengjie Li, "Robust Wireless Statistic Division Multiplexing and Its Performance Analysis", International Journal of Distributed Sensor Network, vol. 14, no. 12, pp. 1-13, 2018

[15] Yanwu Ding, Timothy N. Davidson, Zhi-Quan Luo, et al., "Minmum BER Block for Zero-Forcing Equalization", IEEE Trans. on Signal Processing, vol.51, no.9, pp. 2410-2423, 2003 


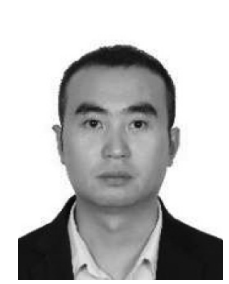

Zhongqiang Luo received the B.S. and M.S. degrees in communication engineering and pattern recognition and intelligent systems from Sichuan University of Science and Engineering, Zigong, China, in 2009 and 2012, respectively. He received the Ph.D. degree in communication and information systems from University of Electronic Science and Technology of China (UESTC), in 2016. Since 2017, he has been with the Sichuan University of Science and Engineering, where he is currently a lecturer. From December 2018-December 2019, he is a visiting scholar with Department of Computer Science and Electrical Engineering in University of Maryland Baltimore County (UMBC). His research interests include machine learning, blind source separation, signal processing for wireless communication system and intelligent signal processing.

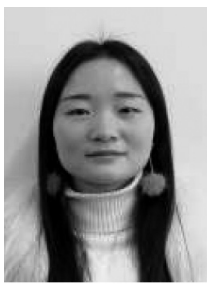

Wei Zhang received her bachelor's degree in electrical engineering and automation from Sichuan University of Science and Engineering(SUSE), Zigong, China, in 2017. Now she is studying at the Sichuan University of Science and Engineering master's degree. Her research interests include blind source separation, signal processing for power line communication and intelligent information processing.

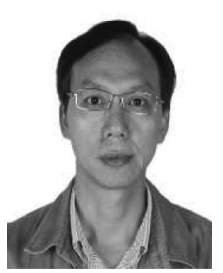

Lidong Zhu received the B.S and M.S from Sichuan University, Chengdu, China, in 1990 and 1999, respectively, and the Ph.D. degree in Communication and Information Systems from Electronic Science and Technology of China (UESTC), Chengdu, China, in 2003. From July 2009 to June 2010, he was a visiting scholar with Department of Electronic Engineering of The University of Hong Kong. His research interests include satellite communications, blind source separation, and communication countermeasure.

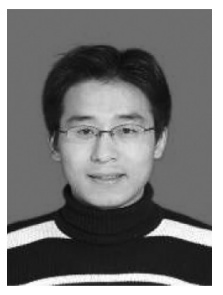

Chengjie Li received the B.Sc. degree in Shandong Normal University, Qufu (Confucius's hometown), China, 2004, the M.Sc. degree in computer software and theory in Xihua University, Chengdu, China, 2009. He received the Ph.D. degree in Communication and Information System in University of Electronic Science and Technology of China (UESTC), Chengdu, China, in 2017. Since 2017, he has been with the Southwest Minzu University, where he is currently a lecturer. His research interests include blind source separation, data mining and intelligent information processing. 